\title{
SINGLE AVERAGE MAGNETIZATION, STAGGERED SYMMETRY, AND NEXT NEAREST NEIGHBORS IN DILUTE SYSTEMS
}

\author{
S. Galam
}

Département de Recherches Physiques, T. 22-23, Université Pierre et Marie Curie, 75252 Paris Cedex 05, France

\begin{abstract}
The usual mean field treatment of dilute antiferromagnets in a uniform field considers two sublattice magnetizations and yields a continuous transition. On the opposite, starting with one single average magnetization produces a tricritical point. This apparent discrepancy is explained by showing that a single magnetization approach does indeed account for the staggered symmetry but also simultaneously includes the effects of next nearest neighbor interactions which were not present in the initial Hamiltonian. The results are discussed with respect to the random field Ising model.
\end{abstract}

The nature of the phase diagram of the Random Field Ising Model (RFIM) has been quite a challenge for theorists over a decade. After a long controversy an exact proof [1] showed that small random fields do not destroy the ferromagnetic long range order of the ground state. Experimentally random fields are produced through either random exchange interactions [2] or site dilution [3]. As yet, must experiments are done using site diluted antiferromagnets [3]. It is therefore of importance to study the Dilute Ising Antiferromagnet in a Field (DIAF).

Monte Carlo (MC) simulations at the threedimensional DIAF at a concentration $p=0.3$ of nonmagnetic sites [4] suggested a transition always continuous. More recently a MC study of the same model but at $p=0.2$ [5] found a tricritical point once next nearest neighbor (nnn) interactions are included. On this basis systematic MC simulations [6] showed that the phase diagram of the DIAF is not universal. It depends on values of both the dilution $p$ and the ratio of $\mathrm{nnn}$ to nearest neighbor (nn) interactions.

In parallel a proper mean field treatment of the DIAF which considers two sublattice magnetizations [7] yields no tricritical point ( $\mathrm{nnn}$ are not included in the DIAF). On the opposite a tricritical point is found when only one single average magnetization is used [8].

In this note it is shown that to consider one single magnetization does take into account the staggered symmetry of the DIAF but simultaneously includes also the effects of nnn which are not present in the initial Hamiltonian [9].

We start with the Hamiltonian for the DIAF

$$
\mathcal{H}=J \sum_{\langle i, j\rangle}^{\mathrm{nn}} \varepsilon_{i} \varepsilon_{j} S_{i} S_{j}-H \sum_{j} \varepsilon_{i} S_{i},
$$

where $J>0, S_{i}= \pm 1, \varepsilon_{i}=1$ with probability $p$, and $\varepsilon_{i}=0$ with probability $(1-p)$. To introduce nnn interactions we add

$$
\mathcal{H}^{\prime}=-\alpha J \sum_{\langle i, j\rangle}^{\mathrm{nnn}} \varepsilon_{i} \varepsilon_{j} S_{i} S_{j},
$$

to $\mathcal{H}$ to get the total Hamiltonian

$$
\mathcal{H}_{\mathrm{T}}=\mathcal{H}+\mathcal{H}^{\prime}
$$

To perform a classical mean field treatment of equation (3) requires the introduction of two sublattices $A$ and $B$ to account for the staggered symmetry of the problem [7]. The equations of state for the total magnetization $m=\frac{1}{2}\left(M_{\mathrm{A}}+M_{\mathrm{B}}\right)$ and the staggered magnetization $M_{\alpha}=\frac{1}{2}\left(M_{\mathrm{A}}-M_{\mathrm{B}}\right)$ which is the order parameter are

$$
M_{\alpha}=\frac{1}{2} p\left(\operatorname{th}\left(\beta H_{\mathrm{A}}\right)-\operatorname{th}\left(\beta H_{\mathrm{B}}\right)\right),
$$

and

$$
m=\frac{1}{2} p\left(\operatorname{th}\left(\beta H_{\mathrm{A}}\right)+\operatorname{th}\left(\beta H_{\mathrm{B}}\right)\right),
$$

where

$$
H_{\mathrm{A}, \mathrm{B}}=H \pm \gamma M_{\alpha}-\delta m
$$

with

$$
\gamma=p(c J+z \alpha J) \text { and } \delta=p(c J-z \alpha J) .
$$

The coordination numbers for $\mathrm{nn}$ and $\mathrm{nnn}$ are respectively $c$ and $z ; \beta=\frac{1}{k_{\mathrm{B}} T}$. At this stage the equation of state for the DIAF is obtained by setting $\alpha=0$ in equation (7) to get $M_{0}$ through equation (4).

Going back to the DIAF we now perform a mean field treatment of equation (1) which considers only one single average magnetization $M^{\prime}$ [8]. The corresponding equation of state is

$$
\begin{aligned}
& M^{\prime}=\frac{1}{2} p\left(\operatorname{th}\left(\beta c J M^{\prime}+\beta H\right)\right.+ \\
&\left.+\operatorname{th}\left(\beta c J M^{\prime}-\beta M\right)\right) .
\end{aligned}
$$

$M^{\prime}$ and $M_{0}$ are rather different. In particular equation (8) yields a tricritical point [8] while equation (4) with $\alpha=0$ does not. On this basis it is tempting to conclude that equation ( 8 ) is wrong since it ignore ap- 
parently the difference between the two sublattices $A$ and $B$ when a field is applied. However it turns out that equation (8) does indeed account for the main physical features of the problem. In fact comparing now equation (4) to equation (8) it appears that for $\delta=0$, i.e.,

$$
\alpha=\frac{c}{z}
$$

Equation (4) becomes

$$
\begin{aligned}
M_{\alpha=\frac{c}{z}}=\frac{1}{2} p(\operatorname{th}(2 \beta c J M & +\beta H)+ \\
& +\operatorname{th}(2 \beta c J M-\beta H)),
\end{aligned}
$$

which is exactly the form of equation (8). The unique difference being the doubling of the coupling constant. This difference is however unimportant since it mainly modifies the value of the Curie temperature. The essential features of the phase diagram, in particular the existence of a tricritical point, are preserved.

In thus appears that the equation of state (8) obtained from equation (1) goes beyond the staggered symmetry by considering also a virtual $\mathcal{H}^{\prime}$ (Eq. (2)). However it is done by taking one particular of $\alpha$ namely $\alpha=c / z$. A study of equations (4) and (5) shows that the existence of a tricritical point depends indeed on the value of $\alpha[7]$. In the range $\alpha>0.6 \mathrm{c} / \mathrm{z}$, a tricritical point occurs while none is found when $\alpha<0.6 \mathrm{c} / z$. On this basis it appears that equation (8) produces a tricritical point since the value $\alpha=c / z$ belongs to the "tricritical range". Therefore the phase diagram obtained for $\alpha=c / z$ is representative for the whole range of ratio $\alpha>0.6 \mathrm{c} / \mathrm{z}$.
In conclusion we compare the above results to those associated with the RFIM. In that case the ground state being ferromagnetic it is totally proper to consider only one single average magnetization. However for the RFIM such a treatment does not induce the inclusion of virtual nnn. Theses differences seem to indicate that the RFIM and the DIAF do not describe the same physical reality. At this stage more rigorous work would clarify the situation.

\section{Acknowledgements}

I would like to thank H. T. Diep and P. Azaria for stimulating discussions.

[1] Imbrie, J., Phys. Rev. Lett. 53 (1984) 1747.

[2] Fisham, S. and Aharony, A., J. Phys. C 12 (1979) L729.

[3] Wong, P. z., Von Molnar, S. and Dimon, P., J. Appl. Phys. 53 (1982) 7954; Solid State Commun. 48 (1983) 573.

[4] Ogielski, A. T. and Huse, D. A., Phys. Rev. Lett. 56 (1986) 1298.

[5] Diep, H. T., Galam, S. and Aziaria, P., Europhys. Lett. 4 (1987) 1067.

[6] Galam, S., Azaria, P. and Diep, H. T., preprint (1988).

[7] Kincaid, J. M. and Cohen, E. G. D., Phys. Rep. 22 (1975) 57.

[8] Galam, S., Phys. Lett. A 121 (1987) 459.

[9] Galam, S., Mod. Phys. Lett. B 1 (1987) 217. 